\title{
Femtosecond coherent anti-Stokes Raman scattering (fs-CARS) for temperature and concentration measurements on combustion species using a dual output OPCPA
}

\author{
Yang $\operatorname{Ran}^{1}$, Marita Kerstan ${ }^{1}$, Andreas Tünnermann ${ }^{1,2}$, Stefan Nolte ${ }^{1,2}$, and Roland \\ Ackermann ${ }^{1, *}$ \\ ${ }^{1}$ Institute of Applied Physics, Abbe Center of Photonics, Albert-Einstein-Straße 15, 07745 Jena, \\ Germany \\ ${ }^{2}$ Fraunhofer Institute for Applied Optics and Engineering (IOF), Albert-Einstein-Straße 7, 07745 \\ Jena, Germany
}

\begin{abstract}
Using two beam femtosecond coherent anti-Stokes Raman scattering (fs-CARS), temperature and concentration measurements are performed on relevant combustion species such as $\mathrm{H}_{2}, \mathrm{~N}_{2}, \mathrm{CO}$, and $\mathrm{CO}_{2}$.
\end{abstract}

\section{Introduction}

Simultaneous temperature and concentration determination is of great importance for applications in gasification [1] and combustion [2]. In this regards, femtosecond coherent anti-Stokes Raman scattering (fs-CARS) is a suitable approach, in particular for high pressure and high temperature conditions [3]. Furthermore, two beam fs-CARS, using a few-cycle pulse for Raman excitation, in principle, allows determining gas temperatures and concentrations of multiple species from a single spectrum $[4,5]$. In this study, we use an optical parametric chirped pulse amplifier (OPCPA) to provide both a broadband $\sim 7 \mathrm{fs}$ pump/Stokes pulse and $\mathrm{a} \sim 1$ ps probe pulse.

\section{Experimental Setup}

The experimental setup is shown in Fig. 1. The two outputs of an OPCPA (venteon OPCPA, Laser Quantum GmbH, Hannover, Germany) are used for the pump/Stokes pulse (650 nm $1100 \mathrm{~nm}, 10 \mu \mathrm{J}, 200 \mathrm{kHz})$, and the probe pulse $(515 \mathrm{~nm}, 20 \mu \mathrm{J}, 200 \mathrm{kHz})$. These two beams are overlapped using a long pass dichroic mirror (LPDM) and then focused into the gas reactor using a concave mirror $(\mathrm{CM})$. This reactor is designed for high temperature and high pressure environment with optical ports made of sapphire [6]. Afterwards, the CARS signal is separated from the residual pump/Stokes and probe pulses with a LPDM and is focused into the spectrometer. The modelling for determining the gas temperature and concentration

* Corresponding author: roland.ackermann@uni-jena.de 
from the CARS spectra is described in detail elsewhere [5, 7]. In this study, experiments were performed on the combustion relevant species $\mathrm{H}_{2}, \mathrm{~N}_{2}, \mathrm{CO}$, and $\mathrm{CO}_{2}$.

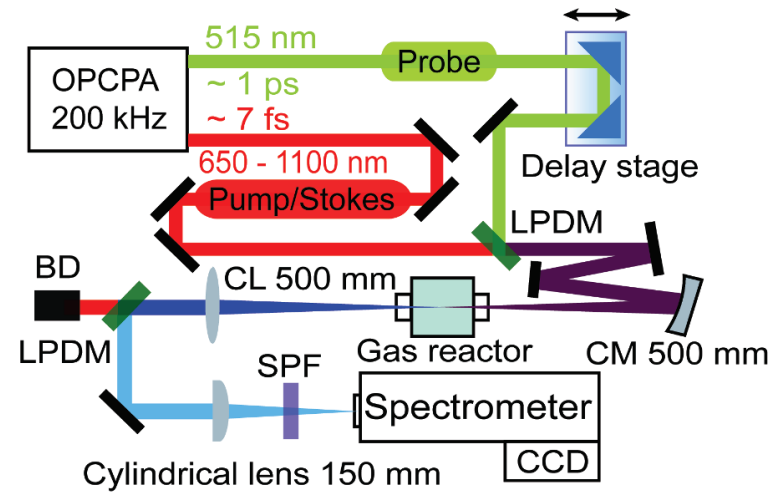

Fig. 1. Fs-CARS experimental setup. LPDM: long pass dichroic mirror; CM: concave mirror; CL: collimating lens; SPF: short pass filter.

\section{Temperature and concentration measurements}

Fig. 2 shows experimental and simulated fs-CARS spectra for concentration determination. Fig. 2(a) shows the fs-CARS spectrum of $2 \% \mathrm{CO}$ in a $\mathrm{CO}$ and $\mathrm{CO}_{2}$ gas mixture at a temperature of $1073 \mathrm{~K}$ and a pressure of 1.4 bar. The best fit of the simulation yields a $\mathrm{CO}$ concentration of $1.8 \%$. Fig. 2(b) shows the fs-CARS spectrum of a gas mixture of multiple species with $7 \% \mathrm{H}_{2}, 55 \% \mathrm{~N}_{2}$, and $38 \% \mathrm{CO}_{2}$ at $478 \mathrm{~K}$ and 1.4 bar, which covers Raman shifts from $\sim 1200 \mathrm{~cm}^{-1}$ to $\sim 4180 \mathrm{~cm}^{-1}$. The corresponding best fits are: $9 \% \mathrm{H}_{2}, 45 \% \mathrm{~N}_{2}$, and $46 \% \mathrm{CO}_{2}$. The deviation between experimental concentration and simulated results is probably mainly influenced by the inhomogeneity of the pump/Stokes spectrum, and will be analysed in future work.
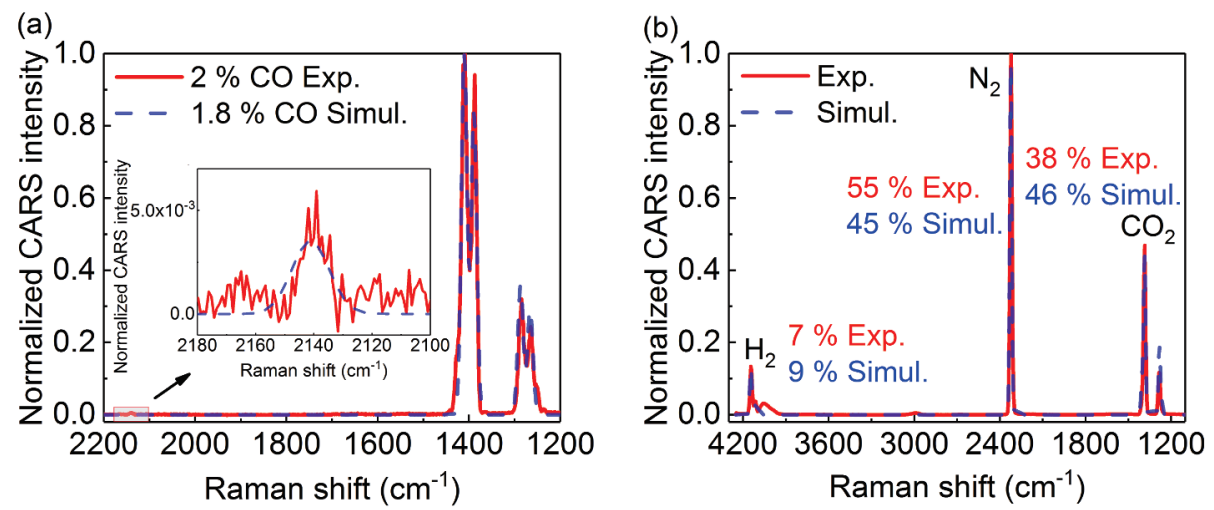

Fig. 2. Experimental and simulated fs-CARS spectra for concentration determination for (a) $2 \% \mathrm{CO}$ (simulated $1.8 \%$ ) in a $\mathrm{CO}-\mathrm{CO}_{2}$ gas mixture at $1073 \mathrm{~K}$ and 1.4 bar (b) gas mixture of $7 \% \mathrm{H}_{2}, 55 \% \mathrm{~N}_{2}$, and $38 \% \mathrm{CO}_{2}$ (simulated $9 \%, 45 \%$ and $46 \%$, respectively, for $\mathrm{H}_{2}, \mathrm{~N}_{2}$ and $\mathrm{CO}_{2}$ ) at $478 \mathrm{~K}$ and 1.4 bar.

For $\mathrm{CO}_{2}$, we have shown recently that the gas temperature can be determined from a single spectrum by means of the temperature dependent population of the vibrational levels [7]. Here, we show that a similar approach is feasible for $\mathrm{H}_{2}$. Due to the high values of the spectroscopic rotational and vibrational constants of $\mathrm{H}_{2}$, the Q-branch CARS lines are widely spaced [8] and are therefore at least partly resolved by our ps probe pulse. 
Fig. 3 shows the corresponding fs-CARS spectra of $\mathrm{H}_{2}$ at $296 \mathrm{~K} / 478 \mathrm{~K}$ with simulated temperature of $323 \mathrm{~K} / 528 \mathrm{~K}$. The Q-branch Q(0), Q(1) and Q(2) lines are apparently hidden within the first large peak at $\sim 4151 \mathrm{~cm}^{-1}$, whereas the $\mathrm{Q}(3)$ peak can be clearly identified. The pronounced background covering $3950 \mathrm{~cm}^{-1}$ to $4100 \mathrm{~cm}^{-1}$ range comes from the pump/Stokes beam of the OPCPA. Future work will try to filter out this background and optimize the probe pulse duration to improve the accuracy of the temperature measurement.

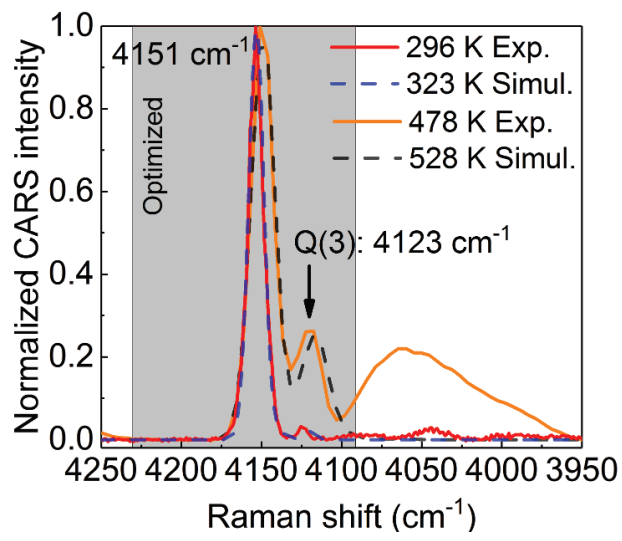

Fig. 3. Experimental and simulated fs-CARS spectrum for temperature determination based on $\mathrm{H}_{2}$ at $296 \mathrm{~K}$ (simulated $323 \mathrm{~K}$ ) and $478 \mathrm{~K}$ (simulated $528 \mathrm{~K}$ ) of $1.4 \mathrm{bar}$. The shadowed grey area shows the optimization range for temperature determination.

\section{Conclusions}

To summarize, two beam fs-CARS using a dual output OPCPA allows concentration measurements in a wide temperature range for the Raman shifts of the most relevant combustion species. For temperature measurements, we detected the ro-vibrational fs-CARS spectrum of $\mathrm{H}_{2}$, and proved its feasibility for temperature determination based on a single spectrum.

This work is supported by the German Federal Ministry of Education and Research (Project OPTICON, Grant-ID: 03Z1H535).

\section{References}

1. C. Higman, S. Tam, Chem. Rev. 114, 1673-708 (2014)

2. S. Roy, J. R. Gord, A. K. Patnaik, Prog. Energy Combust. Sci. 36, 280-306 (2010)

3. R. P. Lucht, S. Roy, T. R. Meyer, J. R. Gord, Appl. Phys. Lett. 89, 251112 (2006)

4. A. Bohlin, C. J. Kliewer, Appl. Phys. Lett. 104, 031107 (2014)

5. G. Matthäus, S. Demmler, M. Lebugle, F. Küster, J. Limpert, A. Tünnermann, S. Nolte, R. Ackermann, Vib. Spectrosc. 85, 128-133 (2016)

6. F. Küster, P. Nikrityuk, M. Junghanns, S. Nolte, A. Tünnermann, R. Ackermann, A. Richter, S. Guhl, B. Meyer, Fuel 194, 544-556 (2017)

7. M. Kerstan, I. Makos, S. Nolte, A. Tünnermann, R. Ackermann, Appl. Phys. Lett. 110, 021116 (2017)

8. V. A. Shakhatov, O. De Pascale, M. Capitelli, K. Hassouni, G. Lombardi, A. Gicquel, Phys. Plasmas 12, 023504 (2005) 